\title{
Linear Logic and Lazy Computation
}

\author{
J.Y. Girard \\ Equipe de Logique, UA753 du CNRS \\ Mathématiques, Tour $45-55,5^{e}$ étage \\ 2 place Jussieu, 75251 Paris CEDEX 05 FRANCE \\ Y. Lafont \\ INRIA, Projet Formel \\ Domaine de Voluceau, Rocquencourt \\ BP105 78153 Le Chesnay CEDEX \\ FRANCE
}

\begin{abstract}
Recently, J.Y. Girard discovered that usual logical connectors such as $\Rightarrow$ (implication) could be broken up into more elementary linear connectors. This provided a new linear logic [Girard86] where hypothesis are (in some sense) used once and only once. The most surprising is that all the power of the usual logic can be recovered by means of recursive logical operators (connector "of course").

There are two versions of the linear logic: the intuitionistic one and the classical one. It seems that the second provides a appropriate formalism for parallelism and communication. This approach is entirely new and requires a further development. Here we restrict our attention to the intuitionistic version and to the consequences of the linear constraint to the computation process.
\end{abstract}

We give two equivalent presentations of the (propositional part of) linear logic: a sequent calculus and a (categorical) combinator system.

Then we introduce inductive and projective connectors, in particular the connector ! (read "of course"). It plays a fundamental role in the encoding of usual intuitionistic logic into linear logic.

There is a cut elimination theorem for the sequent calculus that corresponds to an evaluation mechanism for the combinator system. We present a very simple (abstract) machine that performs linear computations with the following features:

- A very natural lazy evaluation mechanism.

- No need of garbage collector.

Finally, we discuss the relevance of linear logic to implement functional languages. 


\section{A sequent calculus for the linear intuitionistic logic}

First, we present the elementary part of the linear intuitionistic logic in a 'Gentzen like' formalism [Gentzen].

The connectors are 1 (tensor unit), $\otimes$ (tensor product), $\mathbf{t}$ (direct unit), \& (direct product), 0 (direct zero), $\oplus$ (direct sum) and - (linear implication). Thus there are two different conjunctions (the tensor product and the direct product).

In the following rules $A, B, C$ denote formulas and $\Gamma, \Delta$ denote sequences $A_{1}, \ldots, A_{n}$ of formulas. A sequent $A_{1}, \ldots, A_{n} \vdash A$ means that $A$ is a consequent of $A_{1} \otimes \ldots \otimes A_{n}$.

\subsection{Structural rules}

$$
\overline{A \vdash A} \text { (identity) } \frac{\Gamma \vdash A \Delta, A \vdash B}{\Gamma, \Delta \vdash B} \text { (cut) } \frac{\Gamma, A, B, \Delta \vdash C}{\Gamma, B, A, \Delta \vdash C \text { (exchange) }}
$$

\subsection{Logical rules}

$$
\begin{array}{lccc}
\overline{\vdash \mathbf{1}} & \frac{\Gamma \vdash A}{\Gamma, \mathbf{1} A} & \frac{\Gamma \vdash A \Delta \vdash B}{\Gamma, \Delta \vdash A \otimes B} & \frac{\Gamma, A, B \vdash C}{\Gamma, A \otimes B \vdash C} \\
\overline{\Gamma \vdash t} & \frac{\Gamma \vdash A \Gamma \vdash B}{\Gamma \vdash A \& B} & \frac{\Gamma, A \vdash C}{\Gamma, A \& B \vdash C} & \frac{\Gamma, B \vdash C}{\Gamma, A \& B \vdash C} \\
\overline{\Gamma, 0 \vdash A} & \frac{\Gamma \vdash A}{\Gamma \vdash A \oplus B} & \frac{\Gamma \vdash B}{\Gamma \vdash A \oplus B} & \frac{\Gamma, A \vdash C \quad \Gamma, B \vdash C}{\Gamma, A \oplus B \vdash C} \\
& \frac{\Gamma, A \vdash B}{\Gamma \vdash A \rightarrow B} & \frac{\Gamma \vdash A \Delta, B \vdash C}{\Gamma, \Delta, A \rightarrow B \vdash C}
\end{array}
$$

The essential difference with the usual intuitionistic calculus is the absence of two essentially non linear structural rules:

$$
\frac{\Gamma \vdash B}{\Gamma, A \vdash B} \text { (weakening) } \frac{\Gamma, A, A \vdash B}{\Gamma, A \vdash B} \text { (contraction) }
$$

See appendix A for comparison.

Theorem 1 This calculus admits cut elimination: "every proof without hypothesis can be transformed into a cut free proof". 
The proof is essentially the same as Gentzen's one (for usual intuitionistic logic). It is even simpler because of the absence of weakening and contraction.

Let us remind that the cut elimination property has very pleasant consequences: the consistency ${ }^{1}$ of the system and the subformula property (a cut free proof contains only subformulas of the sequent that it proves).

\subsection{Examples of proofs}

From now on, Heyting (logic, formula, proof) means usual intuitionistic.

$A$ first (very crude) interpretation of the system is to see $\mathbf{I}$ and $\mathbf{t}$ as the true proposition, $\otimes$ and $\&$ as a conjunction, 0 as the false proposition, $\oplus$ as a disjunction and $\rightarrow$ as an implication. With this translation, every provable linear formula becomes obviously a provable Heyting formula.

For example, the linear formula $(A \& B) \bullet A$ (here $A$ and $B$ are atomic formulas) has the following (linear) proof:

$$
\frac{\frac{A \vdash A}{A \& B \vdash A}}{\vdash(A \& B) \rightarrow A}
$$

Of course, the corresponding Heyting formula $(A \wedge B) \Rightarrow A$ is also provable.

But the converse is absolutely false: The linear formula $(A \otimes B) \rightarrow A$ is not (linearly) provable. Yet the corresponding Heyting formula $(A \wedge B) \Rightarrow A$ is still provable.

Let us show that $(A \otimes B) \rightarrow A$ is not provable: Take a cut free proof of $\vdash(A \otimes B) \rightarrow A$. The end of your proof has to be:

$$
\frac{\frac{A, B \vdash A}{A \otimes B \vdash A}}{\vdash(A \otimes B) \rightarrow A}
$$

In linear logic, it is impossible to prove $A, B \vdash A$ (in a cut free proof, the last rule has to be an exchange... and you cannot find a beginning for this proof).

One of the notable features of the linear logic is the following distributivity property ( $A \equiv B$ means that $A \vdash B$ and $B \vdash A$ are both provable):

$$
A \otimes(B \oplus C) \equiv(A \otimes B) \oplus(A \otimes C)
$$

Of course it is not true if you replace $\otimes$ by \&.

A cut free proof for the left to right sense is:

$$
\frac{\frac{\overline{A \vdash A} \overline{B \vdash B}}{\frac{A, B \vdash(A \otimes B) \oplus(A \otimes C)}{A, B \otimes B}} \frac{\overline{A \vdash A} \overline{C \vdash C}}{A, C \vdash A \otimes C}}{A, B \oplus C \vdash(A \otimes B) \oplus(A \otimes C)}
$$

Such a cut free proof is easy to find in a bottom up fashion.

\footnotetext{
${ }^{1}$ In our case, the consistency is obvious (it is just a propositional calculus). Moreover there is a very simple translation of the linear logic into the usual one that preserves provability (see section 1.3).
} 


\section{Combinators for the linear logic}

Linear combinators are an alternative presentation for the linear logic ${ }^{2}$.

A combinator is a "name" for an assertion $A \rightarrow B(B$ is consequent of $A$ ), where $A, B$ are formulas. In some sense, combinators are more elementary than sequent rules. Sequent proofs are better for the human, but combinators are closer to the machine.

\subsection{Sequential combinators}

$\overline{\mathrm{id}: A \rightarrow A}$

$$
\frac{x: A \rightarrow B \quad y: B \rightarrow C}{y \circ x: A \rightarrow C}
$$

\subsection{Parallel and arrange combinators}

$$
\overline{\mathbf{1 : 1 \rightarrow 1}}
$$

$\overline{\text { ol }: A \leftrightarrow 1 \otimes A: \mathrm{cl}}$

$\overline{\mathrm{ex}: A \otimes B \leftrightarrow B \otimes A: \mathrm{ex}}$

$$
\frac{x: A \rightarrow B \quad y: C \rightarrow D}{x \otimes y: A \otimes C \rightarrow B \otimes D}
$$

or : $A \leftrightarrow A \otimes \mathbf{1}: \mathbf{c r}$

\subsection{Logical combinators}

$$
\begin{aligned}
& \overline{\langle\rangle: X \rightarrow \mathrm{t}} \quad \frac{x: X \rightarrow A \quad y: X \rightarrow B}{\langle x, y\rangle: X \rightarrow A \& B} \quad \overline{\text { fst }: A \& B \rightarrow A} \quad \overline{\text { snd }: A \& B \rightarrow B} \\
& \overline{\{\}: 0 \rightarrow X} \quad \overline{\operatorname{inl}: A \rightarrow A \oplus B} \quad \overline{\operatorname{inr}: B \rightarrow A \oplus B} \quad \frac{x: A \rightarrow X \quad y: B \rightarrow X}{\{x, y\}: A \oplus B \rightarrow X} \\
& \frac{x: X \otimes A \rightarrow B}{\operatorname{lcur} x: X \rightarrow A \rightarrow B} \quad \overline{\operatorname{lapp}:(A \rightarrow B) \otimes A \rightarrow B}
\end{aligned}
$$

For comparison see appendix B.

Proposition 1 The two formalisms (sequents and combinators) are equivalent:

Every combinator $\varphi: A \rightarrow B$ gives a proof of $A \vdash B$, and every proof of a sequent $A_{1}, \ldots, A_{n} \vdash B$ gives a combinator $\varphi: A_{1} \otimes \ldots \otimes A_{n} \rightarrow B$.

\footnotetext{
${ }^{2}$ They are the exact analogues of what are categorical combinators for Heyting logic (see appendix B) [Lambek80,Curien85, Curien86]. For a category theoretical view, see appendix C.
} 
The proof is straigthforward. In the following rules, $\Gamma$ is cumbersome, but you can push it to the right side using the connector $\rightarrow$ :

$$
\overline{\Gamma, 0 \vdash A} \quad \frac{\Gamma, A \vdash C \Gamma, B \vdash C}{\Gamma, A \oplus B \vdash C}
$$

\section{The connector "of course"}

The linear constraint is very strong. To recover the expressiveness of Heyting logic, it is necessary to introduce a new connector: ! (read "of course").

More generally, we can enrich the linear logic with inductive and projective connectors, two dual notions that we illustrate in the following sections:

\subsection{Inductive connectors}

Let us construct a "type" of natural numbers in our linear logic.

The first solution is a recursive definition (a natural number is zero or the successor of a natural number):

$$
\mathrm{Nat}=\mathbf{1} \oplus \mathrm{Nat}
$$

However, this definition does not capture the fact that Nat is the "best" solution of this "equation". In particular, you need recursive definitions to construct usual functions over integers.

A more adequate solution is to introduce explicit combinators:

$$
\overline{\text { zero: } 1 \rightarrow \text { Nat }} \quad \overline{\text { succ }: \text { Nat } \rightarrow \text { Nat }} \quad \frac{x: 1 \rightarrow X y: X \rightarrow X}{\text { nrec } x y: \text { Nat } \rightarrow X}
$$

Let us give, for example, a (non recursive) definition of the addition:

$$
\begin{aligned}
& \mathrm{cl}: 1 \otimes \mathrm{Nat} \rightarrow \mathrm{Nat} \\
& \longdiv { \text { lcur cl : } 1 \rightarrow \mathrm { Nat } \rightarrow \mathrm { Nat } }
\end{aligned}
$$

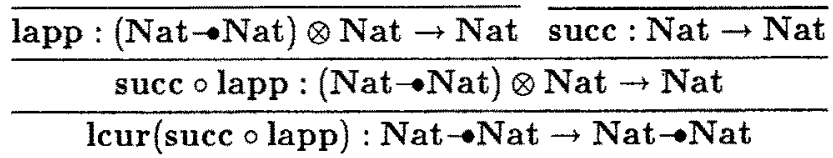

$$
\begin{aligned}
& \text { add }=\text { lapp } \circ((\text { nrec }(\operatorname{lcurcl})(\operatorname{lcur}(\operatorname{succ} \circ \operatorname{lapp}))) \otimes \mathrm{id}): \text { Nat } \otimes \mathrm{Nat} \rightarrow \mathrm{Nat}
\end{aligned}
$$

Other inductive connectors can be introduced, for example the connector List with the following recursive definition:

$$
A \text { List }=\mathbf{1} \oplus(A \otimes(A \text { List }))
$$

The reader may find the corresponding combinators ... 


\subsection{Projective connectors}

If you replace $\oplus$ by \&, you obtain the dual notion of projective connector.

For example the connector! has the following recursive definition:

$$
! A=A \& 1 \&(! A \otimes ! A)
$$

As for Nat, we introduce new combinators:

$$
\frac{x: X \rightarrow A \quad y: X \rightarrow 1 \quad z: X \rightarrow X \otimes X}{\operatorname{make} x y z: X \rightarrow ! A}
$$

$$
\overline{\text { read }: ! A \rightarrow A} \quad \overline{\text { kill }: ! A \rightarrow 1} \quad \overline{\operatorname{dupl}: ! A \rightarrow ! A \otimes ! A}
$$

The connector! is a "trick" to eliminate the linear constraint.

First, $! A$ is a "universal coalgebra" over $A$ :

Proposition 2 The following combinator can be constructed:

$$
\frac{x: ! A \rightarrow B}{\operatorname{lift} x: ! A \rightarrow ! B}
$$

$!$ is also a sort of "exponential" operator (it links together the two conjunctions \& and $\otimes):$

Proposition $3 ! \mathrm{t} \equiv \mathbf{1}$ and $!(A \& B) \equiv ! A \otimes ! B$

In other words, the following combinators can be constructed:

$$
\text { subl }: ! \mathrm{t} \leftrightarrow 1 \text { : crys } \quad \text { crac }: !(A \& B) \leftrightarrow ! A \otimes ! B \text { : glue }
$$

See appendix D for detailed constructions.

\subsection{Encoding of Heyting logic into linear logic}

We saw in section 1.3 a translation of linear logic into Heyting logic. Conversely, Heyting logic can be merged into linear logic (with the connector!).

First we give a translation of Heyting formulas into linear ones:

- $|A|=A(A$ is an atomic formula $)$

- $|t|=\mathrm{t}$ and $|A \wedge B|=|A| \&|B|$

- $|f|=0$ and $|A \vee B|=!|A| \oplus !|B|$

- $|A \Rightarrow B|=!|A| \cdot|B|$

Proposition $4 A$ Heyting formula $A$ is provable if and only if $|A|$ is (linearly) provable. 
The proof uses the following lemma ${ }^{3}$ :

Lemma 1 Let $A, B$ be two Heyting formulas. Every categorical combinator $x: A \rightarrow B$ gives a linear combinator: $|x|:|| A|\rightarrow| B \mid$.

For example, if $x: A \wedge B \rightarrow C$ gives $|x|: !(|A| \&|B|) \rightarrow C$, then cur $x: A \rightarrow B \Rightarrow C$ gives lcur $(|x| \circ$ glue $): !|A| \rightarrow !|B| \rightarrow C$.

In fact, the necessary combinators are exactly those introduced by propositions 2 and 3.

\section{Computation}

\subsection{The evaluation mechanism}

Our purpose is to show that the linear logic is well-suited for lazy evaluation (following the philosophy of [Lafont86]).

Lazy types are:

$$
\text { t } \quad A \& B \quad 0 \quad A \oplus B \quad A \rightarrow B
$$

Values of lazy types are not computed but frozen. A frozen value is made of a constructor and another value, and it is unfrozen by a destructor.

Constructors are:

$$
\langle\langle\varphi, \psi\rangle \text { inl inr } \operatorname{lcur} \varphi
$$

Destructors are:

$$
\text { fst snd }\{\}\{\varphi, \psi\} \text { lapp }
$$

Values are terms:

- 0

- $(u, v)$ where $u, v$ are values

- $\gamma \cdot u$ where $\gamma$ is a constructor and $u$ a value

We inductively define a relation $u: A(u$ "is a value" of $A$ ) for a value $u$ and a linear formula $A$ :

$$
\overline{0: 1} \quad \frac{u: A v: B}{(u, v): A \otimes B} \quad \frac{\gamma: A \rightarrow B \quad u: A}{\gamma \cdot u: B}
$$

We define an operation: $\frac{\varphi: A \rightarrow B \quad u: A}{\varphi u: B}$

\footnotetext{
${ }^{3}$ Note that a Heyting formula $A$ is provable when there exists a categorical combinator $t \rightarrow A$, and a linear formula $A$ is (linearly) provable when there exists a linear combinator $1 \rightarrow A$.
} 
- id $u=u \quad(\varphi \circ \psi) u=\varphi(\psi u)$

- $\mathbf{1}()=0 \quad(\varphi \otimes \psi)(u, v)=(\varphi u, \psi v)$

- ol $u=((), u) \quad \operatorname{cl}((), u)=u$ or $u=(u,()) \quad \operatorname{cr}(u,())=u$ $\operatorname{ex}(u, v)=(v, u)$ al $(u,(v, w))=((u, v), w) \quad \operatorname{ar}((u, v), w)=(u,(v, w))$

- \langle\rangle $u=\langle\rangle \cdot u\langle\varphi, \psi\rangle u=\langle\varphi, \psi\rangle \cdot u$ $\operatorname{fst}(\langle\varphi, \psi\rangle \cdot u)=\varphi u \quad \operatorname{snd}(\langle\varphi, \psi\rangle \cdot u)=\psi u$

- inl $u=\operatorname{inl} \cdot u$ inr $u=\operatorname{inr} \cdot u$ $\{\varphi, \psi\}(\operatorname{inl} \cdot u)=\varphi u \quad\{\varphi, \psi\}(\operatorname{inr} \cdot u)=\psi u$

- $(\operatorname{Icur} \varphi) u=(\operatorname{lcur} \varphi) \cdot u \operatorname{lapp}((\operatorname{lcur} \varphi) \cdot u, v)=\varphi(u, v)$

Theorem 2 The previous definition is well founded: Computations using these rules always terminate.

The proof uses induction over combinators, values and formulas.

The theorem extends to inductive and projective connectors. For example for the connector !:

- $(\operatorname{make} \varphi \psi \rho) u=(\operatorname{make} \varphi \psi \rho) \cdot u$ $\operatorname{read}((\operatorname{make} \varphi \psi \rho) \cdot u)=\varphi u$ $\operatorname{kill}((\operatorname{make} \varphi \psi \rho) \cdot u)=\psi u$ $\operatorname{dupl}((\operatorname{make} \varphi \psi \rho) \cdot u)=((\operatorname{make} \varphi \psi \rho) \otimes(\operatorname{make} \varphi \psi \rho))(\rho u)$

Finally, we may add primitive types with primitive values and primitive combinators, for example a type Num with:

- $0:$ Num $1:$ Num $2:$ Num ...

minus : Num $\rightarrow$ Num sum $:$ Num $\otimes$ Num $\rightarrow$ Num ...

\subsection{The Linear Abstract Machine}

The Linear Abstract Machine is a cousin of the Categorical Abstract Machine [CAM]. But the linear constraint allows a radically different allocation of the memory space.

The memory space is divided into three areas:

- The code area is static (the code doesn't change during the execution) and is organized as a graph.

- The environment area is dynamic with two part: the current tree (or actual environment) and the free list (or memory heap).

- The dump (or stack) is dynamic and linear. 
The main point is that the actual environment is organized as a tree $e^{4}$ and the space allocation is completely provided (no need of garbage collector, see section 5 ).

As usual, the code is a list of elementary instructions (notations: $c:: C$ denotes the list whose head is $c$ and whose tail is $C,[]$ denotes the empty list and $\gamma$ denotes a constructor).

\begin{tabular}{|c|c|c|c|c|c|}
\hline \multicolumn{6}{|c|}{ Linear Abstract Machine } \\
\hline \multicolumn{3}{|c|}{ Before } & \multicolumn{3}{|c|}{ After } \\
\hline code & environment & dump & code & environment & dump \\
\hline$\overline{p u s h l:: C}$ & $(u, v)$ & $D$ & $C$ & $u$ & $v:: D$ \\
\hline consl :: C & $u$ & $v:: D$ & $C$ & $(u, v)$ & $D$ \\
\hline pushr :: $C$ & $(u, v)$ & $D$ & $C$ & $v$ & $u:: D$ \\
\hline consr :: $C$ & $v$ & $u:: D$ & $C$ & $(u, v)$ & $D$ \\
\hline ol ::C & $u$ & $D$ & $\bar{C}$ & $(0, u)$ & $D$ \\
\hline$c l:: C$ & $(0, u)$ & $D$ & $C$ & $u$ & $D$ \\
\hline or $:: C$ & $u$ & $D$ & $C$ & $(u,())$ & $D$ \\
\hline $\operatorname{cr}:: C$ & $(u, 0)$ & $D$ & $C$ & $u$ & $D$ \\
\hline$e x:: C$ & $(u, v)$ & $D$ & $C$ & $(v, u)$ & $D$ \\
\hline$a l:: C$ & $(u,(v, w))$ & $D$ & $C$ & $((u, v), w)$ & $D$ \\
\hline$a r:: C$ & $((u, v), w)$ & $D$ & $C$ & $(u,(v, w))$ & $D$ \\
\hline$\gamma:: C$ & $u$ & $D$ & $C$ & $\gamma \cdot u$ & $D$ \\
\hline fst : $: C$ & $\left(\operatorname{pair}\left(C^{\prime}, C^{\prime \prime}\right)\right) \cdot u$ & $D$ & $C^{\prime}$ & $u$ & $C:: D$ \\
\hline snd :: $C$ & $\left(\operatorname{pair}\left(C^{\prime}, C^{\prime \prime}\right)\right) \cdot u$ & $D$ & $C^{\prime \prime}$ & $u$ & $C:: D$ \\
\hline $\operatorname{altv}\left(C^{\prime}, C^{\prime \prime}\right):: C$ & $i n l \cdot u$ & $D$ & $C^{\prime}$ & $u$ & $C:: D$ \\
\hline $\operatorname{altv}\left(C^{\prime}, C^{\prime \prime}\right):: C$ & $i n r \cdot u$ & $D$ & $C^{\prime \prime}$ & $u$ & $C:: D$ \\
\hline lapp $:: C$ & $\left(\left(\right.\right.$ lcur $\left.\left.C^{\prime}\right) \cdot u, v\right)$ & $D$ & $C^{\prime}$ & $(u, v)$ & $C:: D$ \\
\hline I] & $u$ & $C:: D$ & $C$ & $u$ & $D$ \\
\hline [] & $u$ & [l] & & Return $u$ & \\
\hline
\end{tabular}

Every linear combinator $\varphi$ gives code $\|\varphi\|$ for the LAM (notation: @ denotes the concatenation of lists):

- $\|\mathrm{id}\|=\|\| \varphi \circ \psi\|=\| \psi\|@\| \varphi \|$

- $\|\mathbf{1}\|=\|\mathbf{i d}\|=[]$

$\|\varphi \otimes \psi\|=\|(\mathrm{id} \otimes \psi) \circ(\varphi \otimes \mathrm{id})\|=[$ pushl $] @\|\varphi\| @[$ consl; $p u s h r] @\|\psi\| @[$ consr $]$

For the other connectors, the translation is obvious:

- $\|\langle\varphi, \psi\rangle\|=[\operatorname{pair}(\|\varphi\|,\|\psi\|)] \quad\|\mathrm{fst}\|=[f s t] \ldots$

\subsection{Compilation of inductive and projective combinators}

There is no specific LAM instruction for inductive and projective combinators. In fact they can be compiled into looping code.

Let us consider for example the connector !, with its recursive definition:

${ }^{4}$ In a strong sense, that means a connected graph without cycle and without shared nodes. 


$$
! A=A \& 1 \&(! A \otimes ! A)
$$

!A is a direct product with three projections (Here, trd denotes the third projection):

$$
\text { read }=\text { fst }: ! A \rightarrow A \quad \text { kill }=\text { snd }: ! A \rightarrow 1 \quad \text { dupl }=\operatorname{trd}: ! A \rightarrow ! A \otimes ! A
$$

The combinator make is compiled into the following looping combinator:

make $x$ y $z=m$ where $m=\langle x, y,(m \otimes m) \circ z\rangle$

\section{Relevance of linear logic for computation}

\subsection{Lazyness}

We have to clarify the difference between Heyting logic and linear logic, and the simplification linear logic gives.

In Heyting logic, there is only one conjunction $\wedge$ :

A strict value of $A \wedge B$ is a pair $(u, v)$ where $u$ is a value of $A$ and $v$ a value of $B$. Such a value may be too "evaluated" if you apply the destructor fst or snd.

A lazy value of $A \wedge B$ is a frozen value $\langle\varphi, \psi\rangle \cdot u$ where $u$ is a value of a type $X$ and $\varphi, \psi$ are combinators, $\varphi: X \rightarrow A$ and $\psi: X \rightarrow B$. Such a value may be too little "evaluated" if you apply the destructor app.

Of course, it is possible to unfreeze frozen values when necessary, but this mechanism seems rather complicated and unnatural, compared to the strict evaluation mechanism [CAM,MaSu].

The problem is that two essentially different kinds of destructors (the projections and the application) may operate over values of type $(A \Rightarrow B) \wedge A$.

In linear logic, the dilemma disappears:

Values of $A \otimes B$ are strict values, and the two components are necessary: There is no projection $A \otimes B \rightarrow A$ or $A \otimes B \rightarrow B$.

Values of $A \& B$ are lazy values, and the only possible destructors for such a value are fst and snd.

\subsection{Memory allocation}

Implementations of symbolic (LISP) or functional (ML) languages need a separate mechanism (the garbage collector) to recover the memory space used by abandoned pieces of data. Garbage collecting takes time and sometimes place. Moreover, it complicates the implementation ...

In linear logic, the connector corresponding to the management of environment is $\otimes$ ( $\&$ is lazy). Thus, projections and pairing don't act on the environment. This allows the environment to be kept in a tree whose nodes are never abandoned or shared.

More precisely, the transitions of the Linear Abstract Machine are left and right linear with respect to the environment (but not to the code). Left linearity is expected for an abstract machine, but right linearity is rather surprising. 
In our "implementation", we add a fourth register (the free list) to the Linear Abstract Machine. Some instructions (consl, consr, ol, or and the constructors) take a free location from the free list. Other instructions (pushl, pushr, cl, cr, fst, snd, altv, lapp) return a location to the free list (this is legitimate because nodes are not shared). The other instructions (like $e x$ ) act as physical modifications.

Of course, we don't need a garbage collector because nodes are never abandoned.

\subsection{Compilation of functional languages}

We saw in section 3.3 a translation of categorical combinators into linear combinators. But there is a classical translation of functional programs into categorical combinators [CAM,MaSu]. That gives a compilation of functional programs into the Linear Abstract Machine.

Unfortunately, this compilation is not realistic. In fact, a very simple program gives a big piece of code. For example, the categorical combinator $\varphi \circ \psi$ is translated into the linear combinator $|\varphi| \circ($ lift $|\psi|)$, and lift is not a primitive combinator (see appendix D), and make is not a primitive instruction (see section 4.3 ) ...

Of course, this translation is too brutish. The problem is to understand how the linearity that occurs in a program (and there is a lot of linearity in classical algorithms) can be recognized (by the machine or by the programmer) for our linear implementation.

A possible continuation for this article should be the elaboration of a realistic optimized translation, or rather the development of a new programming style, in a new high level language adapted to our linear implementation. This new language should hold simultaneously elegance of functional languages and efficiency of procedural languages. 


\section{Appendix}

A The sequent calculus for the usual intuitionistic logic

A.1 Structural rules

$$
\begin{array}{cc}
\overline{A \vdash A} \text { (identity) } & \frac{\Gamma \vdash A \Delta, A \vdash B}{\Gamma, \Delta \vdash B} \text { (cut) } \quad \frac{\Gamma, A, B, \Delta \vdash C}{\Gamma, B, A, \Delta \vdash C \text { (exchange) }} \\
\frac{\Gamma \vdash B}{\Gamma, A \vdash B} \text { (weakening) } & \frac{\Gamma, A, A \vdash B}{\Gamma, A \vdash B} \text { (contraction) }
\end{array}
$$

\section{A.2 Logical rules}

$$
\begin{array}{cccc}
\overline{\vdash t} & \frac{\Gamma \vdash A \Delta \vdash B}{\Gamma, \Delta \vdash A \wedge B} & \frac{\Gamma, A \vdash C}{\Gamma, A \wedge B \vdash C} & \frac{\Gamma, B \vdash C}{\Gamma, A \wedge B \vdash C} \\
\overline{f \vdash A} & \frac{\Gamma \vdash A}{\Gamma \vdash A \vee B} & \frac{\Gamma \vdash B}{\Gamma \vdash A \vee B} & \frac{\Gamma, A \vdash C \Delta, B \vdash C}{\Gamma, \Delta, A \vee B \vdash C} \\
& \frac{\Gamma, A \vdash B}{\Gamma \vdash A \Rightarrow B} & \frac{\Gamma \vdash A \Delta, B \vdash C}{\Gamma, \Delta, A \Rightarrow B \vdash C}
\end{array}
$$

\section{B Categorical combinators}

B.1 Sequential combinators

$$
\overline{\text { id : } A \rightarrow A}
$$

$$
\frac{x: A \rightarrow B \quad y: B \rightarrow C}{y \circ x: A \rightarrow C}
$$

\section{B.2 Logical combinators}

$$
\begin{array}{cccc}
\overline{\langle\rangle: X \rightarrow t} & \frac{x: X \rightarrow A y: X \rightarrow B}{\langle x, y\rangle: X \rightarrow A \wedge B} & \overline{\text { fst }: A \wedge B \rightarrow A} & \overline{\text { snd }: A \wedge B \rightarrow B} \\
\overline{\{\}: f \rightarrow X} & \overline{\operatorname{inl}: A \rightarrow A \vee B} & \overline{\operatorname{inr}: B \rightarrow A \vee B} & \frac{x: A \rightarrow X y: B \rightarrow X}{\{x, y\}: A \vee B \rightarrow X} \\
\frac{x: X \wedge A \rightarrow B}{\operatorname{cur} x: X \rightarrow A \Rightarrow B} & \overline{\text { app }:(A \Rightarrow B) \wedge A \rightarrow B}
\end{array}
$$




\section{Linear categories}

\section{C.1 Terminology}

A symmetric monoidal category is a category $C$ with a bifunctor $\theta: C \times C \rightarrow C$ and an object $1 \in C$ such that:

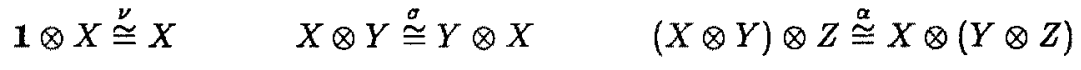

Here $\cong$ denotes a natural isomorphism. In addition, there are several coherence axioms that constrain those natural isomorphims (for example: $\sigma \circ \sigma=\mathrm{id}, \nu \circ \alpha=\nu \otimes \mathrm{id}$ ).

A symmetric monoidal closed category is a symmetric monoidal category $C$ such that, for every $A \in C$, the functor $X \mapsto X \otimes A$ has a right adjoint $Y \mapsto A \rightarrow Y$. That means:

$$
\operatorname{Hom}(X \otimes A, Y) \cong \operatorname{Hom}(X, A \rightarrow Y)
$$

Finally, a linear category is a symmetric monoidal closed category with finite products and coproducts ${ }^{5}$.

\section{C.2 Examples}

Of course, a category with finite products (or coproducts) is a monoidal category. Therefore a category with finite products, finite coproducts and exponentials is a linear category ${ }^{6}$. For example SET is a linear category: $\otimes$ and $\&$ are the cartesian product, $\oplus$ is the disjoint union, and $I \rightarrow J=J^{I}$.

A more interesting model is the category of modules over a ring: $\otimes$ is the tensor product, \& the direct product, $\oplus$ the direct sum, and $A \rightarrow B=\operatorname{Hom}(A, B)$. Of course, \& and $\oplus$ are identical.

Another example is the category TOP of topological spaces. TOP is not cartesian closed but it is a linear category: \& is the cartesian product and $\oplus$ is the disjoint union. $E \otimes F$ is $E \times F$ with the finest topology that makes sections $x \mapsto(x, y)$ and $y \mapsto(x, y)$ continuous. $E \rightarrow F$ is the space of continuous maps $E \rightarrow F$ with the pointwise convergence topology.

\section{Some useful linear combinators}

$$
\begin{gathered}
\text { trans }=\operatorname{ar} \circ((\text { al } \circ(\text { id } \otimes \text { ex }) \circ \text { ar }) \otimes \mathrm{id}) \circ \text { al }:(A \otimes B) \otimes(C \otimes D) \rightarrow(A \otimes C) \otimes(B \otimes D) \\
\frac{x: ! A \rightarrow B \overline{\text { kill }: ! A \rightarrow 1} \overline{\text { dupl }: ! A \rightarrow ! A \otimes ! A}}{\text { lift } x=\text { make } x \text { kill dupl }: ! A \rightarrow ! B}
\end{gathered}
$$

\footnotetext{
${ }^{5}$ The categorical notion corresponding to "!" is more complex. It makes use of the notion of internal comonoid.
} functor). 


$$
\begin{aligned}
& \overline{\text { subl }=\text { kill }: ! \mathrm{t} \rightarrow \mathbf{1}} \\
& \frac{\overline{\langle: 1 \rightarrow \mathrm{t}} \overline{\mathrm{id}: 1 \rightarrow 1} \overline{\text { ol }: 1 \rightarrow(1 \otimes 1)}}{\text { crys }=\text { make }\langle\text { id ol }: 1 \rightarrow ! \mathrm{t}}
\end{aligned}
$$

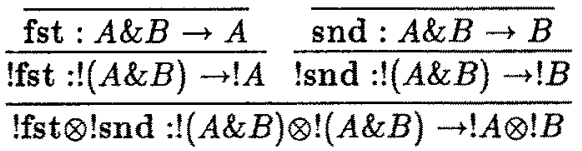

$$
\begin{aligned}
& \text { crac }=(\text { fst } \otimes ! \text { snd }) \circ \text { dupl }:(A \& B) \rightarrow ! A \otimes ! B \\
& \overline{\text { read }: ! A \rightarrow A} \overline{\text { kill }: ! B \rightarrow 1} \\
& \frac{\overline{\text { read } \otimes \text { kill }: ! A \otimes ! B \rightarrow A \otimes 1} \overline{\operatorname{cr}: A \otimes 1 \rightarrow A}}{\operatorname{cr} \circ(\text { read } \otimes \text { kill }): ! A \otimes ! B \rightarrow A} \\
& \overline{\text { kill }: ! A \rightarrow 1} \overline{\text { read }: ! B \rightarrow B} \\
& \frac{\overline{\text { kill } \otimes \text { read }: ! A \otimes ! B \rightarrow 1 \otimes B} \overline{\mathrm{cl}: \mathbf{1} \otimes B \rightarrow B}}{\mathrm{cl} \circ(\text { kill } \otimes \text { read }): ! A \otimes ! B \rightarrow B} \\
& \overline{\text { kill }: ! A \rightarrow 1} \overline{\text { kill }: ! B \rightarrow 1} \\
& \overline{\text { kill } \otimes \text { kill }: ! A \otimes ! B \rightarrow 1 \otimes 1} \overline{\text { cl }: 1 \otimes 1 \rightarrow 1} \\
& \text { cl } \circ(\text { kill } \otimes \text { kill }): ! A \otimes ! B \rightarrow 1 \\
& \overline{\text { dupl }: ! A \rightarrow ! A \otimes ! A} \overline{\text { dupl }: ! B \rightarrow ! B \otimes ! B} \\
& \overline{\text { dupl } \otimes \operatorname{dupl}: ! A \otimes ! B \rightarrow(! A \otimes ! A) \otimes(! B \otimes ! B)}
\end{aligned}
$$

glue $=\operatorname{make}\langle\operatorname{cr} \circ(\operatorname{read} \otimes$ kill $), \operatorname{cl} \circ($ kill $\otimes$ read $)\rangle(\operatorname{cl} \circ($ kill $\otimes$ kill $))($ trans $\circ($ dupl $\otimes$ dupl)) $: ! A \otimes ! B \rightarrow !(A \& B)$

\section{References}

[CAM] G. Cousineau, P.L. Curien and M. Mauny. "The Categorical Abstract Machine." In Functional Programming Languages and Computer Architecture, Ed. J. P. Jouannaud, Springer-Verlag LNCS 201 (1985) 50-64.

[Curien85] P. L. Curien. "Categorical Combinatory Logic." ICALP 85, Nafplion, SpringerVerlag LNCS 194 (1985).

[Curien86] P. L. Curien. "Categorical Combinators, Sequential Algorithms and Functional Programming." Pitman (1986). 
[Gentzen] G. Gentzen. "The Collected Papers of Gerhard Gentzen." Ed. E. Szabo, NorthHolland, Amsterdam (1969).

[Girard86] J.Y. Girard "Linear Logic" to appear in TCS.

[Lafont86] Y. Lafont "De la Déduction Naturelle à Machine Catégorique" to appear.

[Lambek80] J. Lambek. "From Lambda-calculus to Cartesian Closed Categories." in To H. B. Curry: Essays on Combinatory Logic, Lambda-calculus and Formalism, Eds. J. P. Seldin and J. R. Hindley, Academic Press (1980).

[MaSu] M. Mauny and A. Suarez. "Implementing Functional Languages in the Categorical Abstract Machine." in Proceedings of the 1986 ACM Conference on Lisp and Functional Programming. 